\title{
Doublon bottleneck in the ultrafast relaxation dynamics of hot electrons in $1 T-\mathrm{TaS}_{2}$
}

\author{
I. Avigo, ${ }^{1}$ F. Queisser $\odot,{ }^{1,2,3}$ P. Zhou, ${ }^{1}$ M. Ligges,,${ }^{1, *}$ K. Rossnagel, ${ }^{4,5,6}$ R. Schützhold,${ }^{1,2,3, \dagger}$ and U. Bovensiepen ${ }^{1, \$}$ \\ ${ }^{1}$ Fakultät für Physik, Universität Duisburg-Essen, Lotharstrasse 1, 47057 Duisburg, Germany \\ ${ }^{2}$ Helmholtz-Zentrum Dresden-Rossendorf, Bautzner Landstrasse 400, 01328 Dresden, Germany \\ ${ }^{3}$ Institut für Theoretische Physik, Technische Universität Dresden, 01062 Dresden, Germany \\ ${ }^{4}$ Institut für Experimentelle und Angewandte Physik, Christian-Albrechts-Universität zu Kiel, 24098 Kiel, Germany \\ ${ }^{5}$ Ruprecht-Haensel-Labor, Christian-Albrechts-Universität zu Kiel und Deutsches Elektronen-Synchrotron DESY, \\ 24098 Kiel and 22607 Hamburg, Germany \\ ${ }^{6}$ Deutsches Elektronen-Synchrotron DESY, 22607 Hamburg, Germany
}

(Received 26 July 2019; revised manuscript received 7 May 2020; accepted 11 May 2020; published 1 June 2020)

\begin{abstract}
Employing time-resolved photoelectron spectroscopy we analyze the relaxation dynamics of hot electrons in the charge density wave/Mott material $1 T-\mathrm{TaS}_{2}$. At $1.2 \mathrm{eV}$ above the Fermi level we observe a hot electron lifetime of $12 \pm 5 \mathrm{fs}$ in the metallic state and of $60 \pm 10 \mathrm{fs}$ in the broken symmetry ground state-a direct consequence of the reduced phase space for electron-electron scattering determined by the Mott gap. Boltzmann equation calculations which account for the interaction of hot electrons in a Bloch band with a doublon-holon excitation in the Mott state provide insight into the unoccupied electronic structure in the correlated state.
\end{abstract}

DOI: 10.1103/PhysRevResearch.2.022046

The lifetime of an excited, hot electron is determined by the imaginary part of the self-energy and for bulk metals $[1,2]$ and semiconductors [3] a comprehensive understanding has been developed. For materials with strong electron correlations such insight is missing because (i) the electronic structure is considerably more complex and (ii) the excitation of interest may modify the electronic structure hosting it. In recent years established experimental and theoretical approaches, which investigate the thermal equilibrium, were complemented by methods which access nonequilibrium states of matter in the time domain. While part of the activity aims at states and properties which exist out of equilibrium [4-8], also new tools for the analysis of excitations in strongly correlated materials were introduced [9-13]. For low-energy excitations up to $100 \mathrm{meV}$ differences between the single-particle and the population lifetimes occur due to carrier relaxation and multiplication [14]. Such differences are absent for higher energies [15]. Due to the complexity of the problem rather simple models such as the Falicov-Kimball model [16,17], the Hubbard model [18,19], or sophisticated Holstein models [20] were studied so far. Treating an actual femtosecond (fs) laser excitation employed in experimental realizations with optical inter- and/or intraband transitions is challenging [21,22]. An experimental realization of such models is possible in ultra-

\footnotetext{
*Present address: Fraunhofer IMS, 47057 Duisburg, Germany.

†r.schuetzhold@hzdr.de

‡uwe.bovensiepen@uni-due.de
}

Published by the American Physical Society under the terms of the Creative Commons Attribution 4.0 International license. Further distribution of this work must maintain attribution to the author(s) and the published article's title, journal citation, and DOI. cold atomic gases [23,24]. For solid materials they represent only a part of the full problem because delocalized Bloch electrons are not included in the model though they are essential in real materials. Therefore, it is important to treat both the correlated electron states as well as weakly correlated Bloch bands including the interaction between these two electron systems.

In this Rapid Communication we analyze such an interaction of correlated electrons with Bloch electrons. We investigate hot electron relaxation in the charge density wave/Mott material $1 T-\mathrm{TaS}_{2}$ by fs time-resolved photoelectron spectroscopy measurements and Boltzmann equation calculations, which treat the interaction of delocalized, propagating electrons in a Bloch band with the correlated electron system. We explain the up to five times longer hot electron lifetimes observed in the correlated, low-temperature state as compared to the metallic, high-temperature state by a doublon bottleneck in the correlated state. The absence of electronic states in the Mott gap up to an excitation energy set by the Coulomb repulsion $U$ reduces the phase space for electron-electron scattering considerably and increases the hot electron lifetime in the Bloch band. Thus, the observed electron lifetime provides insight into the excited electronic structure in the correlated state, in particular into the interaction strength of Bloch electrons with the strongly correlated electrons.

We perform time-resolved photoelectron spectroscopy on $1 T-\mathrm{TaS}_{2}$ as described earlier [22]. The pump excitation is induced by laser pulses at $1.53 \mathrm{eV}$ photon energy and $50 \mathrm{fs}$ pulse duration, which are generated by regenerative chirped pulse amplification at a $250 \mathrm{kHz}$ repetition rate in a commercial Ti:sapphire amplifier (Coherent RegA 9040). Photoelectrons are created by probe pulses at $6.1 \mathrm{eV}$ photon energy and 100 fs pulse duration, which are obtained by frequency quadrupling in $\beta$-barium borate crystals and analyzed in normal 
emission geometry by an electron time-of-flight spectrometer with $\pm 0.02 \AA^{-1}$ parallel momentum resolution and $50 \mathrm{meV}$ effective energy resolution. The cross-correlation width of pump and probe pulses is measured on the sample surface at maximum electron kinetic energy and is $110 \pm 10 \mathrm{fs}$. Single-crystals of $1 T-\mathrm{TaS}_{2}$ grown as described in detail in Ref. [22] are cleaved in ultrahigh vacuum at a base pressure of $1 \times 10^{-10} \mathrm{mbar}$.

The investigated material $1 T-\mathrm{TaS}_{2}$ is a layered transition metal dichalcogenide which presents a charge density wave (CDW) order of increasing degree of commensurability the lower the temperature $T$ becomes [25]. At $T=370 \mathrm{~K}$ the $\mathrm{CDW}$ is incommensurate and a Fermi-Dirac distribution function at the Fermi energy $E_{\mathrm{F}}$ is observed in photoemission spectroscopy [see Fig. 1(a)], indicating a metallic state. Below $T=180 \mathrm{~K}$, after having passed through a nearly commensurate (metallic) CDW state, the commensurate CDW forms and the Ta atoms rearrange into clusters out of $13 \mathrm{Ta}$ atoms each in a $\sqrt{13} \times \sqrt{13}$ reconstructed, triangular lattice, as depicted in Fig. 1(c). The transition into the commensurate CDW is accompanied by an order of magnitude increase in the electrical resistivity and an increase of spectral weight $150 \mathrm{meV}$ below $E_{\mathrm{F}}$ [see Figs. 1(b) and 1(c) and Refs. [26-28]], indicating a Mott transition of the half-filled CDW subband close to $E_{\mathrm{F}}[25,29]$. This concept has been challenged recently by the proposal of orbital ordering leading to the insulating state [30]. Furthermore, variations in stacking are reported to couple electronic states of adjacent layers [31,32]. The broken symmetry ground states of $1 T-\mathrm{TaS}_{2}$ provide rich timedependent structural and electronic dynamics which have led to various ultrafast experiments which provide manyfold new insights into the complex interplay of lattice and electrons in correlated systems [6,22,26,33-37]. Here, we exploit the opportunity to compare the ultrafast electron dynamics for a metallic state with the CDW/Mott state in a single material. As such $1 T-\mathrm{TaS}_{2}$ serves as a model system to showcase the doublon bottleneck effect, as detailed below.

Figure 1 presents photoelectron spectra on a logarithmic intensity axis for the incommensurate, metallic CDW state at $T=370 \mathrm{~K}$ [Fig. 1(a)] and the commensurate, insulating CDW state at $T=30 \mathrm{~K}$ [Figs. 1(b) and 1(c)] for different pump-probe time delays $t$. In the metallic case the spectra follow a thermalized Fermi-Dirac distribution function at $t \geqslant$ $200 \mathrm{fs}$ as indicated by the dashed lines in Fig. 1(a). With increasing $t$ the respective electron temperature decreases, which is explained by energy transfer to phonons [34]. At earlier delays weak deviations from a thermalized distribution are identified. In the insulating state the spectra exhibit a more involved behavior. Overall, the electron distribution relaxes towards lower energy with increasing $t$. However, no simple description using a thermal distribution as for $370 \mathrm{~K}$ succeeds. Spectra at different $t$ vary weakly up to $E-E_{\mathrm{F}}=0.1 \mathrm{eV}$ and fan out toward higher energy. Until $t=400 \mathrm{fs}$ a significant electron population at $E-E_{\mathrm{F}}=0.5 \mathrm{eV}$ is observed. For later $t$ relaxation towards $E-E_{\mathrm{F}}=0.1 \mathrm{eV}$ is found. These effects are observed for different incident pump fluences $F$ [Figs. 1(b) and 1(c)], and are more intense for higher $F$.

For further analysis we turn to the time-dependent photoelectron intensity as a function of $E-E_{\mathrm{F}}$, because the

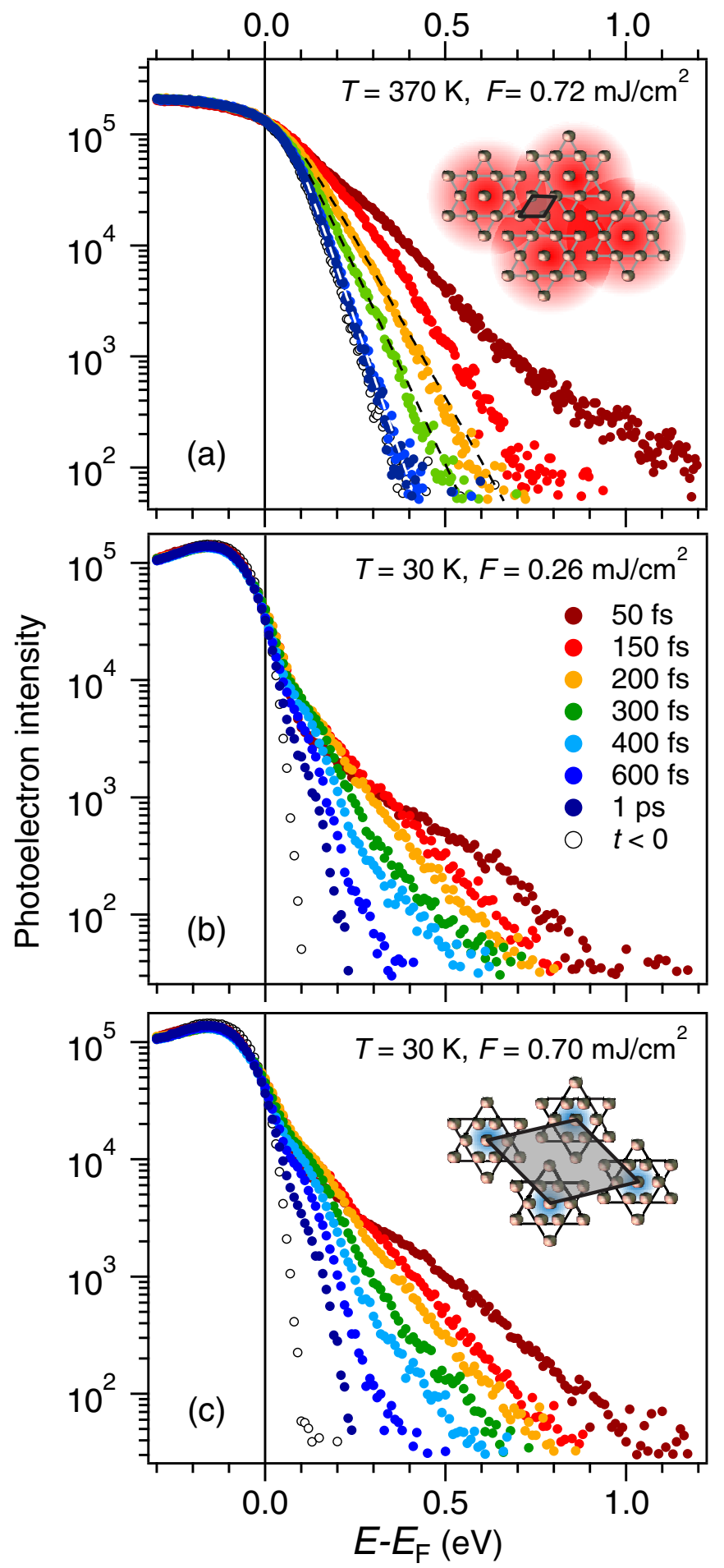

FIG. 1. Time-resolved photoemission spectra detected in normal emission geometry for (a) the metallic and (b), (c) charge density wave/Mott state at the indicated time delay, temperature, and incident pump fluence $F$. Insets depict the in-plane structure of Ta atoms in the metallic and CDW state. Dashed lines in (a) indicate thermalized electron distribution functions at electron temperatures 873,715 , and $524 \mathrm{~K}$. All spectra are referenced to $E_{\mathrm{F}}$ of the metallic state.

electron dynamics in the metallic and insulating states can be analyzed quantitatively in terms of an energy-dependent relax- 

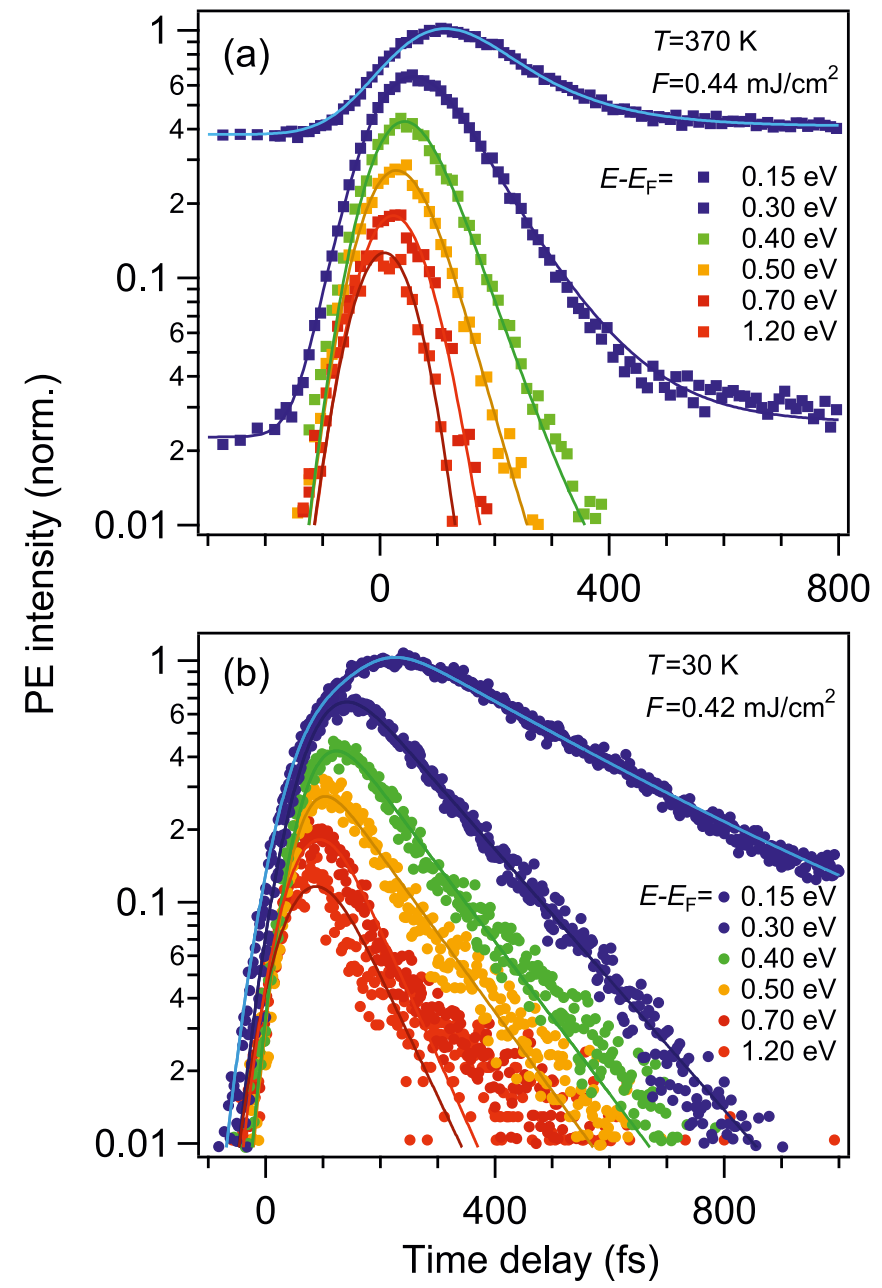

FIG. 2. Photoelectron intensity as a function of time delay at indicated energies for (a) the metallic and (b) the CDW/Mott state. Solid lines represent fits (see text).

ation time $\tau\left(E-E_{\mathrm{F}}\right)$. Figure 2(a) shows the time-dependent photoelectron intensity at $T=370 \mathrm{~K}$, which is characterized by a decreasing $\tau$ with increasing $E-E_{\mathrm{F}}$. The finite intensity for 0.15 and $0.30 \mathrm{eV}$ at $t>1$ ps originates from thermally populated states close to $E_{\mathrm{F}}$. Such qualitative behavior is indeed known for metals because of the increasing phase space for electron-electron scattering for growing $E-E_{\mathrm{F}}$ $[1,2]$. As depicted in Fig. 2(b), $\tau$ decreases with $E-E_{\mathrm{F}}$ also for $T=30 \mathrm{~K}$, however, much weaker than for the metallic case. At $E-E_{\mathrm{F}}=1.2 \mathrm{eV}$, for example, the relaxation is at $30 \mathrm{~K}$ clearly slower than at $370 \mathrm{~K}$. We analyze the electron dynamics by fitting the data to

$$
N(t, E)=\left[\left(N_{0} e^{-t / \tau(E)}+N_{1}\right) \cdot \Theta(t)\right] \otimes g(t)+N_{2} .
$$

Here, $\Theta(t)$ is the Heaviside function, $g(t)$ is a Gaussian function representing the cross correlation width of pump and probe laser pulses, $N_{1}$ is the population at the asymptotic value for $t>0$, and $N_{2}$ is an offset. The latter two are nonzero for metallic states at 0.15 and $0.3 \mathrm{eV}$ [see Fig. 2(a)]. The determined lifetimes $\tau(E)$ are depicted in Fig. 3 for different pump fluences. While for all data sets lower-energy electrons exhibit longer lifetimes than higher-energy electrons,

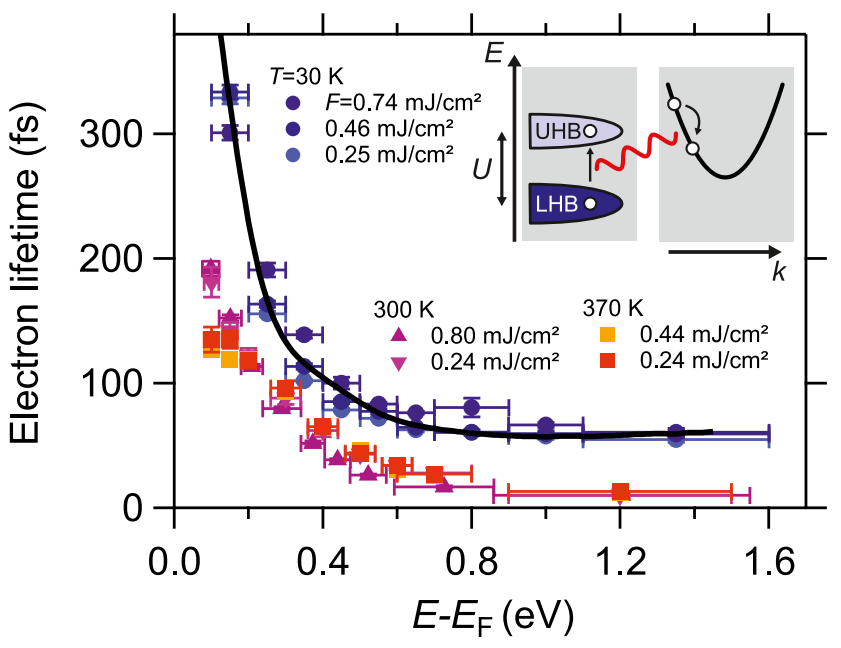

FIG. 3. Hot electron lifetimes $\tau$ as a function of electron energy determined by fitting the time-dependent photoelectron intensities for different equilibrium temperatures as indicated. Horizontal error bars indicate the analyzed, integrated spectral width. The solid line is the result of the Boltzmann equation calculation (see the Supplemental Material [38]). The inset shows the interaction considered in these calculations: Relaxation of a delocalized hot electron in a Bloch band $\mathcal{E}_{\mathbf{k}}$ mediated by excitation of a doublon-holon pair of energy $\approx U$ in the Fermi-Hubbard system.

we identify a systematic dependence in $\tau(E)$ on whether the material is in the metallic state at $T=300$ and $370 \mathrm{~K}$ or in the commensurate $\mathrm{CDW} /$ Mott state at $30 \mathrm{~K}$. In the latter case $\tau(E)$ lies well above the values for the metallic state independent on the chosen $F$. At the highest energy analyzed the determined $\tau$ differ for 30 and $370 \mathrm{~K}$ by a factor of five.

In order to illustrate the distinctive features of the relaxation dynamics, we consider the following simple model (see the Supplemental Material [38]). The cold electrons in the Mott insulator state are described by the creation and annihilation operators $\hat{c}_{\mu, s}^{\dagger}$ and $\hat{c}_{\nu, s}$ with spin $s \in\{\uparrow, \downarrow\}$ at the lattice sites $\mu$ and $\nu$. We model their internal dynamics via the Fermi-Hubbard Hamiltonian [39,40]

$$
\hat{H}_{\mathrm{FH}}=-\sum_{\mu, v, s} J_{\mu \nu} \hat{c}_{\mu, s}^{\dagger} \hat{c}_{\nu, s}+U \sum_{\mu} \hat{n}_{\mu}^{\uparrow} \hat{n}_{\mu}^{\downarrow},
$$

with the hopping matrix $J_{\mu \nu}$, the on-site repulsion $U$, and the particle numbers $\hat{n}_{\mu}^{s}=\hat{c}_{\mu, s}^{\dagger} \hat{c}_{\mu, s}$.

The hot electrons are described by the operators $\hat{a}_{\mu, s}^{\dagger}$ and $\hat{a}_{v, s}$. In view of the observed fluence independence of their relaxation (see Fig. 3), we neglect their interactions among each other and describe their internal dynamics via the free-electron approximation $\hat{H}_{\text {free }}=\sum_{\mathbf{k}, s} \mathcal{E}_{\mathbf{k}} \hat{a}_{\mathbf{k}, s}^{\dagger} \hat{a}_{\mathbf{k}, s}$ with the Bloch band energies $\mathcal{E}_{\mathbf{k}}$. Since the hot electrons are observed not to populate the upper Hubbard band during their relaxation [22], we neglect direct transitions between them and the cold electrons, i.e., we do not include transition terms such as $\hat{a}_{\mu, s}^{\dagger} \hat{c}_{v, s}+$ H.c. (see the Supplemental Material [38]). Nevertheless, there are interactions (e.g., Coulomb) between 
them, as described by

$$
\hat{H}_{\text {int }}=\sum_{\mu, v, s, s^{\prime}} V_{\mu, \nu}^{s, s^{\prime}} \hat{c}_{\mu, s}^{\dagger} \hat{c}_{\mu, s} \hat{a}_{\nu, s^{\prime}}^{\dagger} \hat{a}_{\nu, s^{\prime}}
$$

with the interaction matrix elements $V_{\mu, v}^{s, s^{\prime}}$.

As the total Hamiltonian $\hat{H}=\hat{H}_{\mathrm{FH}}+\hat{H}_{\text {free }}+\hat{H}_{\text {int }}$ cannot be solved exactly, we have to employ suitable approximations. For weak interactions $U$ and $V_{\mu, v}^{s, s^{\prime}}$, one may employ standard perturbation theory. However, this is not possible in the strongly correlated Mott state, so we use the method of the hierarchy of correlations instead (see, e.g., Refs. [41-46]). To this end, we start with a mean-field approximation of the Mott state $\hat{\varrho}_{\mu} \approx(|\uparrow\rangle\langle\uparrow|+| \downarrow\rangle\langle\downarrow|) / 2$ without spin ordering, because we have a triangular lattice which is not bipartite and thus prevents antiferromagnetic ordering due to spin frustration. Note that we consider the propagation and interaction of the hot and cold electrons within a single layer, i.e., we treat them within a two-dimensional (2D) model (see the Supplemental Material [38]).

Following a strategy analogous to Refs. [47,48], we may derive the Boltzmann equation describing the evolution of the distribution functions $f_{\mathbf{k}, s}=\left\langle\hat{a}_{\mathbf{k}, s}^{\dagger} \hat{a}_{\mathbf{k}, s}\right\rangle$ of the hot electrons due to their interaction with the cold electrons in the Mott insulator state,

$$
\begin{aligned}
\partial_{t} f_{\mathbf{k}, s}= & -\sum_{s^{\prime}} \int_{\mathbf{p}} \int_{\mathbf{q}}\left|V_{\mathbf{q}}^{s, s^{\prime}}\right|^{2} M_{\mathbf{p}, \mathbf{q}} \delta\left(\mathcal{E}_{\mathbf{k}}-\mathcal{E}_{\mathbf{k}-\mathbf{q}}-E_{\mathbf{p}+\mathbf{q}}^{+}+E_{\mathbf{p}}^{-}\right) \\
& \times\left[f_{\mathbf{k}, s}\left(1-f_{\mathbf{k}-\mathbf{q}, s}\right)\left(1-f_{\mathbf{p}+\mathbf{q}, s^{\prime}}^{+}\right)\left(1-f_{\mathbf{p}, s^{\prime}}^{-}\right)-\text {inverse }\right] .
\end{aligned}
$$

The integrals over momenta $\mathbf{p}$ and $\mathbf{q}$ cover the entire Brillouin zone. As usual, $V_{\mathbf{q}}^{s, s^{\prime}}$ is the Fourier transform of the Coulomb interaction matrix $V_{\mu, v}^{s, s^{\prime}}$ evaluated at the momentum transfer q. The above channel describes the inelastic scattering of a hot electron from initial $\mathbf{k}$ to final momentum $\mathbf{k}-\mathbf{q}$ while creating a doublon-holon pair with momenta $\mathbf{p}+\mathbf{q}$ and $\mathbf{p}$, described by their distribution functions $f_{\mathbf{p}+\mathbf{q}, s^{\prime}}^{+}$and $f_{\mathbf{p}, s^{\prime}}^{-}$as well as the matrix elements $M_{\mathbf{p}, \mathbf{q}}$, plus the inverse process. The delta function in Eq. (4) corresponds to energy conservation with the doublon and holon excitation energies

$$
E_{\mathbf{p}}^{ \pm}=\frac{1}{2}\left(U-J_{\mathbf{p}} \pm \sqrt{J_{\mathbf{p}}^{2}+U^{2}}\right),
$$

where $J_{\mathbf{p}}$ is the Fourier transform of the matrix $J_{\mu \nu}$.

Motivated by the experimentally observed, ultrashort doublon lifetimes [22], we neglect preexisting doublon-holon excitations in the Mott insulator state, such that the above relaxation channel (4) is dominant. Thus, we may employ the standard relaxation time approximation $f_{\mathbf{k}-\mathbf{q}, s} \approx 0, f_{\mathbf{p}+\mathbf{q}, s^{\prime}}^{+} \approx$ 0 , and $f_{\mathbf{p}, s^{\prime}}^{-} \approx 0$ which gives $\partial_{t} f_{\mathbf{k}, s}=-f_{\mathbf{k}, s} / \tau_{\mathbf{k}}$ (see the Supplemental Material [38]). As further approximations, we describe the energies of the hot electrons by a parabolic dispersion $\mathcal{E}_{\mathbf{k}} \approx \mathcal{E}_{0}+\mathbf{k}^{2} /\left(2 m_{*}\right)$ with the effective mass $m_{*}$ and assume that the interaction matrix $V_{\mu, \nu}^{s, s^{\prime}}$ is dominated by the local (on-site) term $V_{\mu, \nu}^{s, s^{\prime}} \approx V \delta_{\mu \nu}$ in analogy to (2). Under these assumptions we may calculate the relaxation time $\tau_{\mathbf{k}}$. The qualitative $\mathbf{k}$ dependence of $\tau_{\mathbf{k}}$ can be understood in terms of phase-space arguments. As a peculiarity of two spatial dimensions, the energy $\mathcal{E}_{\mathbf{k}-\mathbf{q}}$ and the "volume" factor of the $\mathbf{q}$ integral in (4) are both quadratic in q. Thus, they effectively cancel each other and $\tau_{\mathbf{k}}$ becomes approximately independent of $\mathbf{k}$ for large energies, i.e., large $\mathbf{k}$.

For the strongly interacting limit $U \gg J$ (i.e., deep within the Mott insulating phase), we may further approximate $E_{\mathbf{p}+\mathbf{q}}^{+}-E_{\mathbf{p}}^{-} \approx U$. Then we find that the plateau of the relaxation rate $\tau_{\mathbf{k}}$ at high energies roughly scales with $V^{2} m_{*} \ell^{2} J^{2} / U^{2}$, where $\ell$ is the lattice spacing [i.e., the distance between neighboring "stars of David" in Fig. 1(c)]. For smaller energies, however, the available phase space shrinks and eventually vanishes: If the initial energy $\mathcal{E}_{\mathbf{k}}$ of the hot electron becomes too small to create a doublon-holon pair, i.e., $\mathbf{k}^{2} /\left(2 m_{*}\right)<U$, the channel (4) closes and $1 / \tau_{\mathbf{k}}$ vanishes. These phase-space arguments yield an approximate step-function behavior of $1 / \tau_{\mathbf{k}}$. Decay of a hot electron in the Bloch band is mediated by excitation of a doublon-holon pair, and this coupling imposes a bottleneck for the relaxation (see Fig. 3). There will be further relaxation channels due to coupling to phonons which will result in a nonzero relaxation rate $1 / \tau_{\mathbf{k}}$ at energies below $U$ leading to lattice heating as observed experimentally [34].

In order to arrive at a more quantitative comparison, we have to specify the relevant model parameters such as $J$ and $U$, etc. Unfortunately, their precise value is still a somewhat open question, so we assume potentially realistic values of $U=0.35 \mathrm{eV}$ and $J=0.05 \mathrm{eV}$ as a working hypothesis (cf. Ref. [22]). The Bloch band $\mathcal{E}_{\mathbf{k}} \approx \mathcal{E}_{0}+\mathbf{k}^{2} /\left(2 m_{*}\right)$ is described by the two parameters $\mathcal{E}_{0}$ and $m_{*}$. Since photoelectrons are observed down to quite low energies relative to $E_{\mathrm{F}}$, we assume $\mathcal{E}_{0}=0$ [40]. Other values of $\mathcal{E}_{0}$ would just shift the curve $\tau(E)$ horizontally and thus do not affect the height of the plateau at high energies. However, the effective mass $m_{*}$ does also affect the functional form of $\tau(E)$ as well as the plateau height - remember the rough scaling law $1 / \tau_{\mathbf{k}} \sim$ $V^{2} m_{*} \ell^{2} J^{2} / U^{2}$ described above. In view of the delocalized and quasifree nature of the hot electrons, as obtained in density functional theory calculations in coexistence with the strongly correlated electrons [49], we assume $m_{*}=m_{e}$. Then, using the approximation $V_{\mu, v}^{s, s^{\prime}} \approx V \delta_{\mu \nu}$ mentioned above, we may obtain the remaining unknown parameter $V$ by fitting the energy-dependent relaxation rate $\tau(E)$, especially the plateau height (see Fig. 3), which gives $V \approx 0.085 \mathrm{eV}$.

A value of $V$ below $U$ is quite natural as $U$ is determined by the Coulomb overlap integral between the same charge density distributions (up to the opposite spin) of the cold electrons in the Mott state while $V$ corresponds to the overlap between the charge density distributions of the cold and the hot (Bloch band) electrons. At small energies, the fit in Fig. 3 should be taken cum grano salis because there low-energy relaxation channels such as coupling to phonons (which we have not included here) may become important. Nevertheless, the result $V<U$ obtained from the plateau at high energies is quite robust, unless extremely small values of $m_{*}$ are assumed. This coupling $V$ shows that the Fermi-Hubbard model [Eq. (2)] alone is not sufficient for a complete description and should also be important for understanding other phenomena, such as optical conductivity, charge carrier multiplication, doublon-holon recombination, and the potential suitability of this material for Mott transistor applications [50]. 
In conclusion, we have observed hot electron decay in a strongly correlated electron material to exhibit at high energy $\mathcal{E}>U$ a rather long lifetime, which we assign to its coupling to a secondary doublon-holon excitation acting as a bottleneck in the decay. A model description which considers coupling of delocalized, hot Bloch electrons with the doublon-holon excitation provides a description of the excited electronic structure, which we expect to have a considerable impact in the field of strongly correlated electron materials in general.

We acknowledge fruitful discussions with Peter Kratzer and other members of the SFB 1242. This work was funded by the Deutsche Forschungsgemeinschaft (DFG), Grant No. 278162697 (SFB 1242).
[1] E. V. Chulkov, A. G. Borisov, J. P. Gauyacq, D. Sánchez-Portal, V. M. Silkin, V. P. Zhukov, and P. M. Echenique, Chem. Rev. 106, 4160 (2006).

[2] M. Bauer, A. Marienfeld, and M. Aeschlimann, Prog. Surf. Sci. 90, 319 (2015).

[3] J. Shah, Ultrafast Spectroscopy of Semiconductors and Semiconductor Nanostructures, 2nd ed. (Springer, Berlin, 1999).

[4] H. Ichikawa, S. Nozawa, T. Sato, A. Tomita, K. Ichiyanagi, M. Chollet, L. Guerin, N. Dean, A. Cavalleri, S.-i. Adachi et al., Nat. Mater. 10, 101 (2011).

[5] M. Först, C. Manzoni, S. Kaiser, Y. Tomioka, Y. Tokura, R. Merlin, and A. Cavalleri, Nat. Phys. 7, 854 (2011).

[6] L. Stojchevska, I. Vaskivsky, T. Mertelj, D. Svetin, S. Brazovskii, and D. Mihailovic, Science 344, 177 (2014).

[7] X. Cui, C. Wang, A. Argondizzo, S. Garrett-Roe, B. Gumhalter, and H. Petek, Nat. Phys. 10, 505 (2014).

[8] D. N. Basov, R. D. Averitt, and D. Hsieh, Nat. Mater. 16, 1077 (2017).

[9] Dynamics at Solid State Surfaces and Interfaces, edited by U. Bovensiepen, H. Petek, and M. Wolf, Vol. 1 (Wiley-VCH, Berlin, 2012).

[10] C. Giannetti, M. Capone, D. Fausti, M. Fabrizio, F. Parmigiani, and D. Mihailovic, Adv. Phys. 65, 58 (2016).

[11] S. Gerber, S.-L. Yang, D. Zhu, H. Soifer, J. A. Sobota, S. Rebec, J. J. Lee, T. Jia, B. Moritz, C. Jia et al., Science 357, 71 (2017).

[12] H. Aoki, N. Tsuji, M. Eckstein, M. Kollar, T. Oka, and P. Werner, Rev. Mod. Phys. 86, 779 (2014).

[13] S. Parham, H. Li, T. J. Nummy, J. A. Waugh, X. Q. Zhou, J. Griffith, J. Schneeloch, R. D. Zhong, G. D. Gu, and D. S. Dessau, Phys. Rev. X 7, 041013 (2017).

[14] S.-L. Yang, J. A. Sobota, D. Leuenberger, Y. He, M. Hashimoto, D. H. Lu, H. Eisaki, P. S. Kirchmann, and Z.-X. Shen, Phys. Rev. Lett. 114, 247001 (2015).

[15] K. Boger, M. Roth, M. Weinelt, T. Fauster, and P.-G. Reinhard, Phys. Rev. B 65, 075104 (2002).

[16] M. Eckstein and M. Kollar, Phys. Rev. Lett. 100, 120404 (2008).

[17] B. Moritz, A. F. Kemper, M. Sentef, T. P. Devereaux, and J. K. Freericks, Phys. Rev. Lett. 111, 077401 (2013).

[18] M. Moeckel and S. Kehrein, Phys. Rev. Lett. 100, 175702 (2008).

[19] M. Eckstein, M. Kollar, and P. Werner, Phys. Rev. Lett. 103, 056403 (2009).

[20] M. Sentef, A. F. Kemper, B. Moritz, J. K. Freericks, Z.-X. Shen, and T. P. Devereaux, Phys. Rev. X 3, 041033 (2013).

[21] J. D. Rameau, S. Freutel, A. F. Kemper, M. A. Sentef, J. K. Freericks, I. Avigo, M. Ligges, L. Rettig, Y. Yoshida, H. Eisaki et al., Nat. Commun. 7, 13761 (2016).
[22] M. Ligges, I. Avigo, D. Golež, H. U. R. Strand, Y. Beyazit, K. Hanff, F. Diekmann, L. Stojchevska, M. Kalläne, P. Zhou et al., Phys. Rev. Lett. 120, 166401 (2018).

[23] R. Jördens, N. Strohmaier, K. Günter, H. Moritz, and T. Esslinger, Nature (London) 455, 204 (2008).

[24] T. Esslinger, Annu. Rev. Condens. Matter Phys. 1, 129 (2010).

[25] B. Sipos, A. F. Kusmartseva, A. Akrap, H. Berger, L. Forro, and E. Tutis, Nat. Mater. 7, 960 (2008).

[26] L. Perfetti, P. A. Loukakos, M. Lisowski, U. Bovensiepen, H. Berger, S. Biermann, P. S. Cornaglia, A. Georges, and M. Wolf, Phys. Rev. Lett. 97, 067402 (2006).

[27] K. Rossnagel, J. Phys.: Condens. Matter 23, 213001 (2011).

[28] I. Avigo, P. Zhou, M. Kalläne, K. Rossnagel, U. Bovensiepen, and M. Ligges, Appl. Sci. 9, 44 (2019).

[29] P. Fazekas and E. Tosatti, Philos. Mag. B 39, 229 (1979).

[30] T. Ritschel, J. Trinckauf, K. Koepernik, B. Buchner, M. v. Zimmermann, H. Berger, Y. I. Joe, P. Abbamonte, and J. Geck, Nat. Phys. 11, 328 (2015).

[31] A. S. Ngankeu, S. K. Mahatha, K. Guilloy, M. Bianchi, C. E. Sanders, K. Hanff, K. Rossnagel, J. A. Miwa, C. Breth Nielsen, M. Bremholm et al., Phys. Rev. B 96, 195147 (2017).

[32] S.-H. Lee, J. S. Goh, and D. Cho, Phys. Rev. Lett. 122, 106404 (2019).

[33] J. Demsar, L. Forró, H. Berger, and D. Mihailovic, Phys. Rev. B 66, 041101(R) (2002).

[34] M. Eichberger, H. Schäfer, M. Krumova, M. Beyer, J. Demsar, H. Berger, G. Moriena, G. Sciaini, and R. J. D. Miller, Nature (London) 468, 799 (2010).

[35] S. Hellmann, M. Beye, C. Sohrt, T. Rohwer, F. Sorgenfrei, H. Redlin, M. Kalläne, M. Marczynski-Bühlow, F. Hennies, M. Bauer et al., Phys. Rev. Lett. 105, 187401 (2010).

[36] J. C. Petersen, S. Kaiser, N. Dean, A. Simoncig, H. Y. Liu, A. L. Cavalieri, C. Cacho, I. C. E. Turcu, E. Springate, F. Frassetto, L. Poletto, S. S. Dhesi, H. Berger, A. Cavalleri et al., Phys. Rev. Lett. 107, 177402 (2011).

[37] S. Vogelgesang, G. Storeck, J. G. Horstmann, T. Diekmann, M. Sivis, S. Schramm, K. Rossnagel, S. Schäfer, and C. Ropers, Nat. Phys. 14, 184 (2017).

[38] See Supplemental Material at http://link.aps.org/supplemental/ 10.1103/PhysRevResearch.2.022046 for motivation and further details on the employed modeling.

[39] J. Hubbard, Proc. R. Soc. London, Ser. A 276, 238 (1963).

[40] Actually, we have several energy reference scales in our setup: the Fermi energy $E_{\mathrm{F}}$, the upper and lower Hubbard bands from Eq. (2), the minimum $\mathcal{E}_{0}$ of the Bloch band, and the energy scale 
labeling the observed photoelectrons. As explained above, the latter is calibrated with respect to $E_{\mathrm{F}}$. Here, we use the convention $E_{\mathrm{F}}=0$ such that the lower Hubbard band from Eq. (2) is just below $E_{\mathrm{F}}=0$ (due to the finite hopping rate $J=0.05 \mathrm{eV}$ ) while the upper Hubbard band is well above $E_{\mathrm{F}}=0$ (due to the on-site repulsion $U=0.35 \mathrm{eV}$ ) [see Eq. (5)]. This choice is consistent with the Mott insulator picture and motivated by the measured spectra, such as in Fig. 1(b), where we observe a peak at $E-E_{\mathrm{F}} \approx-0.15 \mathrm{eV}$, which is absent in the metallic phase [see Fig. 1(a)]. Thus, we interpret this peak as a signature of the lower Hubbard band following Refs. [26,27] and references therein. The upper Hubbard band can be observed at very short times and positive energies around $E-E_{\mathrm{F}}=0.18 \mathrm{eV}$ (see, e.g., Ref. [22]). Finally, the assumption $\mathcal{E}_{0}=0$ is motivated by the fact that we observe photoelectrons down to quite low energies relative to $E_{\mathrm{F}}$ even within the Hubbard gap. However, one should bear in mind that our method is not too sensitive to this value of $\mathcal{E}_{0}$ because changes of $\mathcal{E}_{0}$ would just shift the curve in Fig. 3 horizontally and thus do not affect the plateau in electron lifetimes observed for $E-E_{\mathrm{F}}>0.6 \mathrm{eV}$ and $T=30 \mathrm{~K}$. Hence, such horizontal shifts would mainly affect the behavior at low energies, where other effects- such as the coupling to phonons - could play a role (but are not included in our analysis).

[41] P. Navez and R. Schützhold, Phys. Rev. A 82, 063603 (2010).

[42] F. Queisser, P. Navez, and R. Schützhold, Phys. Rev. A 85, 033625 (2012).

[43] F. Queisser, K. V. Krutitsky, P. Navez, and R. Schützhold, Phys. Rev. A 89, 033616 (2014).

[44] K. V. Krutitsky, P. Navez, F. Queisser, and R. Schützhold, EPJ Quantum Technol. 1, 12 (2014).

[45] P. Navez, F. Queisser, and R. Schützhold, J. Phys. A: Math. Theor. 47, 225004 (2014).

[46] P. Navez, F. Queisser, and R. Schützhold, Phys. Rev. A 94, 023629 (2016).

[47] F. Queisser and R. Schützhold, Phys. Rev. A 100, 053617 (2019).

[48] F. Queisser, S. Schreiber, P. Kratzer, and R. Schützhold, Phys. Rev. B 100, 245110 (2019).

[49] X.-L. Yu, D.-Y. Liu, Y.-M. Quan, J. Wu, H.-Q. Lin, K. Chang, and L.-J. Zou, Phys. Rev. B 96, 125138 (2017).

[50] G. Mazza, A. Amaricci, M. Capone, and M. Fabrizio, Phys. Rev. Lett. 117, 176401 (2016). 